\title{
Mutual regulation between deubiquitinase CYLD and retroviral oncoprotein Tax
}

Xuefeng $\mathrm{Wu}^{1,3+}{ }^{+}$, Minying Zhang ${ }^{2,3+}$ and Shao-Cong Sun ${ }^{3^{*}}$

\begin{abstract}
Background: Oncoprotein Tax, encoded by the human T-cell leukemia virus type 1 (HTLV1), persistently induces NF- $\kappa$ B activation, which contributes to HTLV1-mediated T-cell transformation. Recent studies suggest that the signaling function of Tax requires its ubiquitination, although how the Tax ubiquitination is regulated remains unclear.

Results: We show here that the deubiquitinase CYLD physically interacts with Tax and negatively regulates the ubiquitination of this viral protein. This function of CYLD is associated with inhibition of Tax-mediated activation of IKK although not that of Tak1. Interestingly, CYLD undergoes constitutive phosphorylation in HTLV1-transformed $T$ cells, a mechanism known to inactivate the catalytic activity of CYLD. Consistently, a phospho-mimetic CYLD mutant fails to inhibit Tax ubiquitination.

Conclusion: These findings suggest that CYLD negatively regulates the signaling function of Tax through inhibition of Tax ubiquitination. Conversely, induction of CYLD phosphorylation may serve as a mechanism by which HTLV1 overrides the inhibitory function of CYLD, leading to the persistent activation of NF- $\kappa \mathrm{B}$.
\end{abstract}

Keywords: CYLD, HTLV, Tax, ubiquitination, IKK, NF-K>B

\section{Background}

Human T-cell leukemia virus type 1 (HTLV1) is an oncogenic retrovirus that is etiologically associated with a human acute $\mathrm{T}$-cell malignancy termed adult $\mathrm{T}$-cell leukemia (ATL) [1-3]. HTLV1 genome encodes a 40-kD protein that not only regulates viral gene expression but also induces various cellular genes contributing to HTLV1-mediated T-cell transformation [4]. Tax modulates the activity of different cellular transcription factors, most importantly NF- $\kappa \mathrm{B}$, a family of enhancer-binding proteins regulating cell growth and survival [5]. The activity of NF- $\kappa \mathrm{B}$ is normally subject to tight regulation by a cytoplasmic inhibitor, $\mathrm{I} \kappa \mathrm{B}$. In response to cellular stimuli, $\mathrm{I} \kappa \mathrm{B}$ is phosphorylated by a specific $\mathrm{I} \kappa \mathrm{B}$ kinase (IKK) and targeted for ubiquitination and proteasomal degradation, resulting in nuclear translocation of active NF- $\kappa \mathrm{B}[6,7]$. Under normal conditions, the activation of IKK and NF- $\kappa \mathrm{B}$ occurs transiently, which assures that

\footnotetext{
* Correspondence: ssun@mdanderson.org

† Contributed equally

${ }^{3}$ Department of Immunology, The University of Texas MD Anderson Cancer Center, 7455 Fannin Street, Box 902, Houston TX 77030, USA

Full list of author information is available at the end of the article
}

the expression of NF- $\kappa \mathrm{B}$ target genes is induced temporally. However, in HTLV1-infected T cells, Tax persistently stimulates the activity of IKK, leading to constitutive nuclear expression of NF- $\kappa \mathrm{B}$ [8-10]. Strong evidence suggests that deregulated NF- $\kappa \mathrm{B}$ activation has a central role in HTLV1-mediated T-cell transformation $[5,11,12]$.

We and others have previously shown that Tax physically interacts with the IKK complex via the IKK regulatory subunit IKK $\gamma$ (also called NEMO), and this molecular interaction is critical for Tax-mediated IKK activation [13-15]. More recent work suggests that the signaling function of Tax requires its ubiquitination [16-18]. Although ubiquitination is traditionally viewed as a mechanism that mediates protein degradation in the proteasome, it is now clear that specific types of ubiquitination also facilitate the activation of protein kinases, including IKK [19]. In particular, lysine 63 (K63)-linked polyubiquitin chains may serve as a platform that helps recruit and activate IKK and its activating kinase, Tak1. Like phosphorylation, ubiquitination is a reversible reaction, which is counter-regulated by ubiquitinating enzymes and deubiquitinases (DUBs) [20]. A DUB, CYLD, has been

\section{() Biomed Central}


shown to preferentially deconjugate K63-linked ubiquitin chains [21] and implicated as a negative regulator of IKK/ NF- $\kappa$ B signaling. CYLD has constitutive DUB activity, but its activity can be rapidly inactivated via its phosphorylation in response to NF- $\kappa$ B stimuli [22].

Tax undergos K63 type of ubiquitination, which is critical for activation of NF- $\kappa$ B [23]. However, how the ubiquitination of Tax is regulated remains unclear. In the present study, we have shown that Tax forms a complex with CYLD, in which CYLD strongly inhibits the ubiquitination and signaling function of Tax. Interestingly, in a large panel of HTLV1-transformed T-cell lines, CYLD is constitutively phosphorylated. These findings not only establish CYLD as a negative regulator of Tax ubiquitination but also suggest a mutual regulatory mechanism in which HTLV1 stimulates CYLD phosphorylation and functional inactivation.

\section{Results}

\section{Tax physically interacts with CYLD}

A prior study suggests that Tax is preferentially conjugated with K63-linked ubiquitin chains [23]. Since CYLD is a K63-specific DUB, we examined whether the ubiquitination of Tax is negatively regulated by CYLD. We first examined the potential physical interaction between Tax and CYLD. In HTLV1-transformed T cells, Tax was readily co-precipitated with CYLD, suggesting that CYLD is present in the Tax complex (Figure 1A). The Tax/CYLD physical association is specific, since a pre-immune serum did not precipitate Tax (Figure 1A). Furthermore, Tax also interacted with CYLD in transiently transfected cells (Figure 1B). Interestingly, the Tax/CYLD interaction appeared to be enhanced by the IKK regulatory subunit, IKK $\gamma$, which is known to interact with both Tax [13-15] and CYLD $[24,25]$. The Tax/CYLD association was also suggested by their colocalization in the cytoplasm of the transfected cells (Figure 1C).

\section{CYLD inhibits Tax ubiquitination}

We next examined whether CYLD regulates the ubiquitination of Tax. As expected, Tax was constitutively ubiquitinated when expressed in 293 cells (Figure 2A). The ubiquitin chains conjugated to Tax appeared to be predominantly K63-linked, since a ubiquitin mutant lacking K63 (K63R) was defective in mediating Tax ubiquitination, whereas the K48R ubiquitin mutant was competent in mediating Tax ubiquitination (Figure 2B, lanes 1 and 2). Furthermore, a ubiquitin mutant retaining lysine 63 but none of the other lysines (K63) was able to mediate Tax ubiquitination (Figure 2B, lane 4). Since CYLD is a DUB that specifically deubiquitinates K63 ubiquitin chains [21,24-26], we tested whether the ubiquitination of Tax is subject to regulation by CYLD. Interestingly, the ubiquitination of Tax was strongly inhibited when it was coexpressed with CYLD (Figure 2C). The inhibitory effect of CYLD on Tax ubiquitination was dependent on its DUB catalytic activity, since a catalytically inactive CYLD mutant failed to inhibit Tax ubiquitination (Figure 2C). These data indicate that CYLD functions as a DUB that negatively regulates Tax ubiquitination.

\section{CYLD inhibits Tax-stimulated activation of IKK but not that of Tak1}

IKK activation by cellular signals is known to require the MAP3K Tak1 [27-32]. Tak1-mediated phosphorylation of IKK $\beta$, together with a ubiquitin-dependent mechanism, regulate IKK activation by the TCR/CD28 signals [33]. We have previously shown that Tax stimulates the catalytic activity of both Tak1 and IKK [34], although the underlying mechanism remains unclear. Since CYLD deubiquitinates Tax, we examined the effect of CYLD on these Tax-specific signaling events. When expressed in 293 cells, Tak 1 displayed a low level of basal catalytic activity as determined by in vitro kinase assay (Figure 3, panel 1). As expected, the kinase activity of Tak1 was potently stimulated by Tax, which was associated with activation of its downstream kinase IKK (Figure 3, panels 1 and 3). Importantly, expression of wildtype CYLD, but not its catalytically inactive mutant, strongly inhibited Tax-stimulated activation of IKK (Figure 3, panel 3), supporting a role for Tax ubiquitination in the activation of IKK signaling. However, to our surprise, CYLD did not affect Tax-stimulated activation of Tak1. Thus, ubiquitination is differentially required for Tax-mediated activation of Tak1 and IKK. This finding is also consistent with our previous observation that Tax-mediated Tak1 activation is required but not sufficient for IKK activation [34].

\section{CYLD is constitutively phosphorylated in HTLV1- transformed T cells}

The catalytic activity of CYLD is negatively regulated by its phosphorylation [22]. In response to cellular signals, CYLD becomes transiently phosphorylated and inactivated, thus contributing to the activation of IKK. Since Tax stimulates persistent activation of IKK, we examined the status of CYLD phosphorylation in a large panel of T-cell lines transformed by HTLV1 or Tax. As expected, the level of $\mathrm{I} \kappa \mathrm{B} \alpha$ (a primary target of IKK) was low in these HTLV1-transformed T cell lines (Figure 4). Remarkably, in all of these Tax-expressing cell lines, CYLD was detected as two bands in IB assays (Figure 4). As previously observed in mitogen-stimulated $\mathrm{T}$ cells, the upper band represented the phosphorylated CYLD, since it was converted to the basal form upon in vitro phosphatase treatment (Figure 4). Thus, CYLD undergoes constitutive phosphorylation in HTLV1-transformed T cells. 

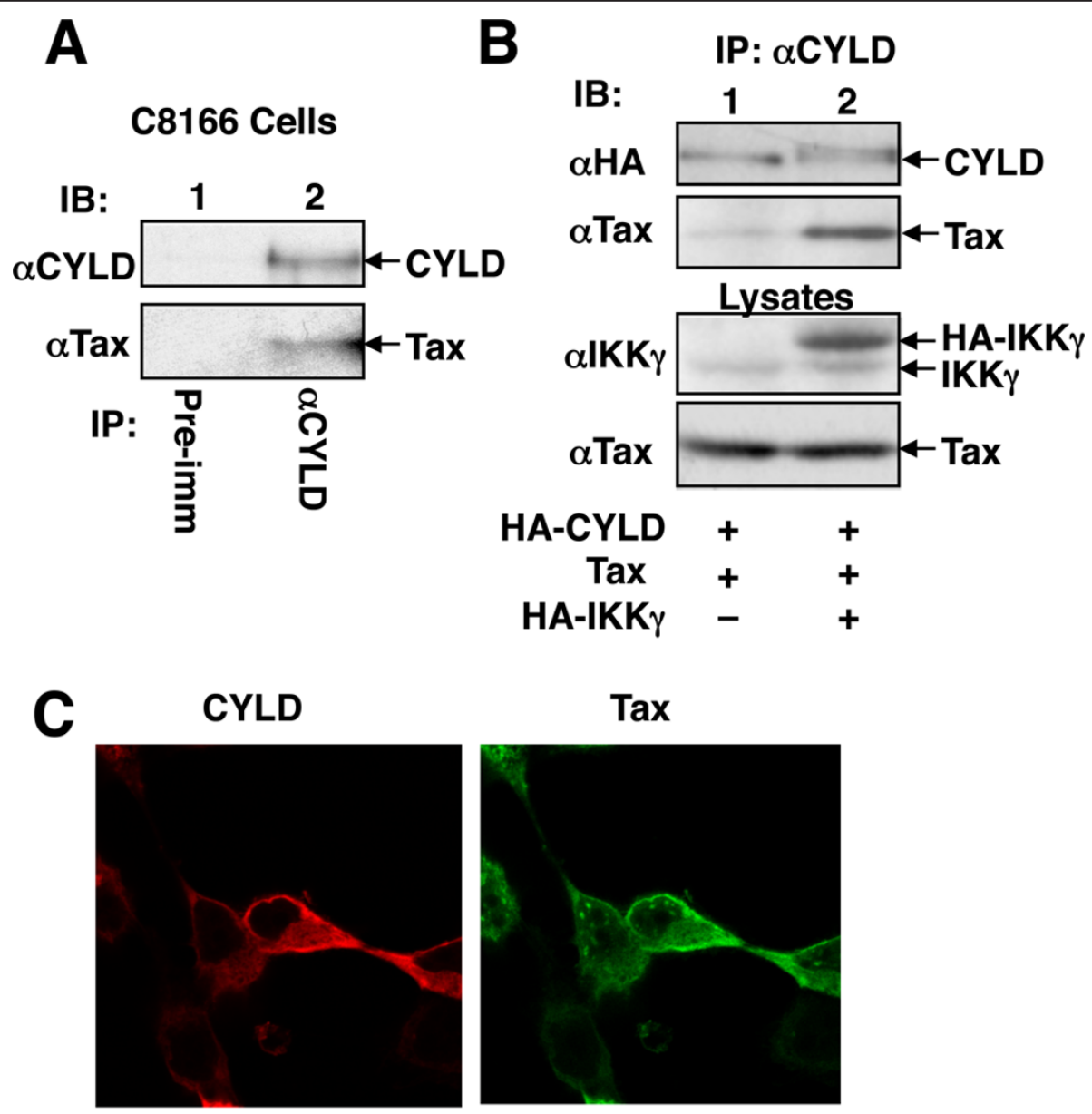

Tax
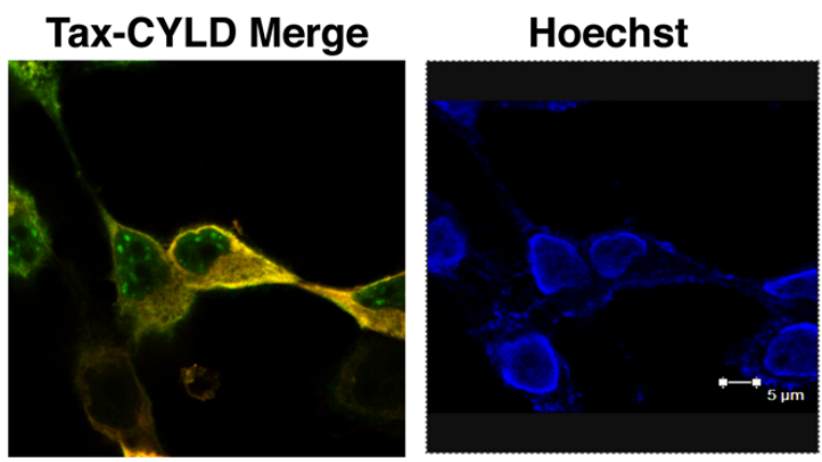

Figure 1 Physical interaction between Tax and CYLD. (A) Cell lysates were prepared from the HTLV1-transformed C8166 cell line and subjected to IP using either a control pre-immune serum or anti-CYLD. The precipitated CYLD and its associated Tax were analyzed by IB. (B) HEK293 cells were transfected with HA-CYLD and Tax either in the absence (-) or presence (+) of IKK $\gamma$. The cell lysates were subjected to IP using anti-CYLD followed by IB to detect the precipitated CYLD and its associated Tax (top two panels). The cell lysates were also subjected to direct IB to monitor the expression of IKK $\gamma$ and Tax. (C) HEK293 cells were transfected with HA-CYLD and Tax. The cells were stained with anti-HA (Y11) and a mouse monoclonal anti-Tax antibody, followed with Texas red-conjugated donkey anti-rabbit Ig and FITC-conjugated donkey antimouse Ig. Cells were also counterstained with Hoechst 33258 for nuclear visualization. The expression of CYLD, Tax, Tax-CYLD merge, and nucleus (Hoechst) are shown. Note that the cytoplasmic, but not nuclear, Tax was colocalized with CYLD (Tax-CYLD merge, yellow color).

A phospho-mimetic CYLD mutant failed to inhibit Tax ubiquitination

To assess the role of CYLD phoshorylation in regulating its catalytic activity, we examined the effect of a phospho-mimetic CYLD mutant on Tax ubiquitination. As expected, the wildtype CYLD, but not its catalytically inactive mutant, efficiently inhibited Tax ubiquitination (Figure 5, lanes 2 and 3). Importantly, a phospho- 


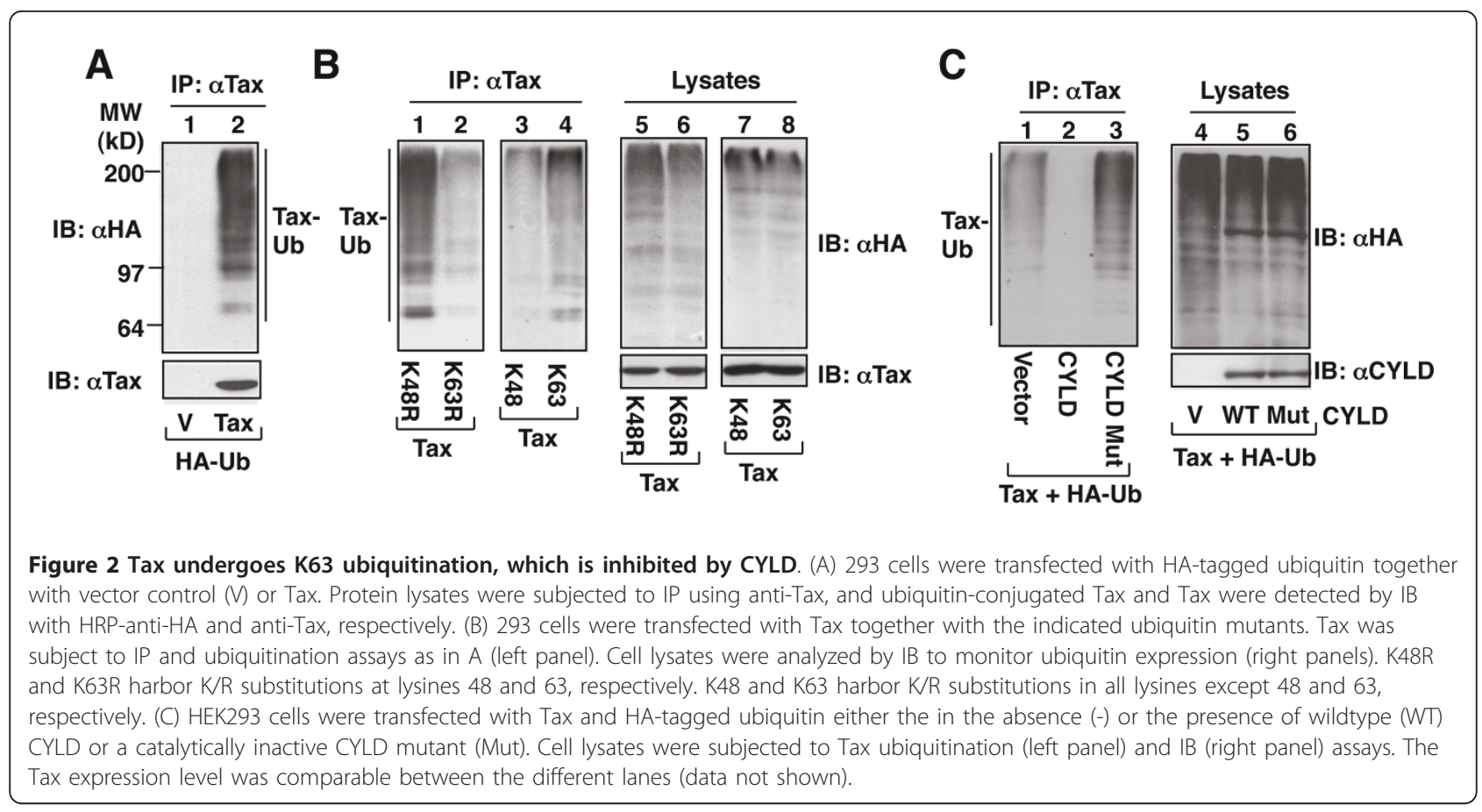

mimetic CYLD mutant harboring serine to glutamic acid substitutions at the phosphorylation sites (CYLD7SE, see ref. [22]) completely failed to deubiquitinate Tax (Figure 5, lane 5), whereas a mutant harboring serine to analine mutations at the phsphorylation sites of CYLD (CYLD7SA) remained active in Tax deubiquitination (Figure 5, lane 4). Thus, in HTLV1-transformed T cells, CYLD is inactivated via constitutive phosphorylation,

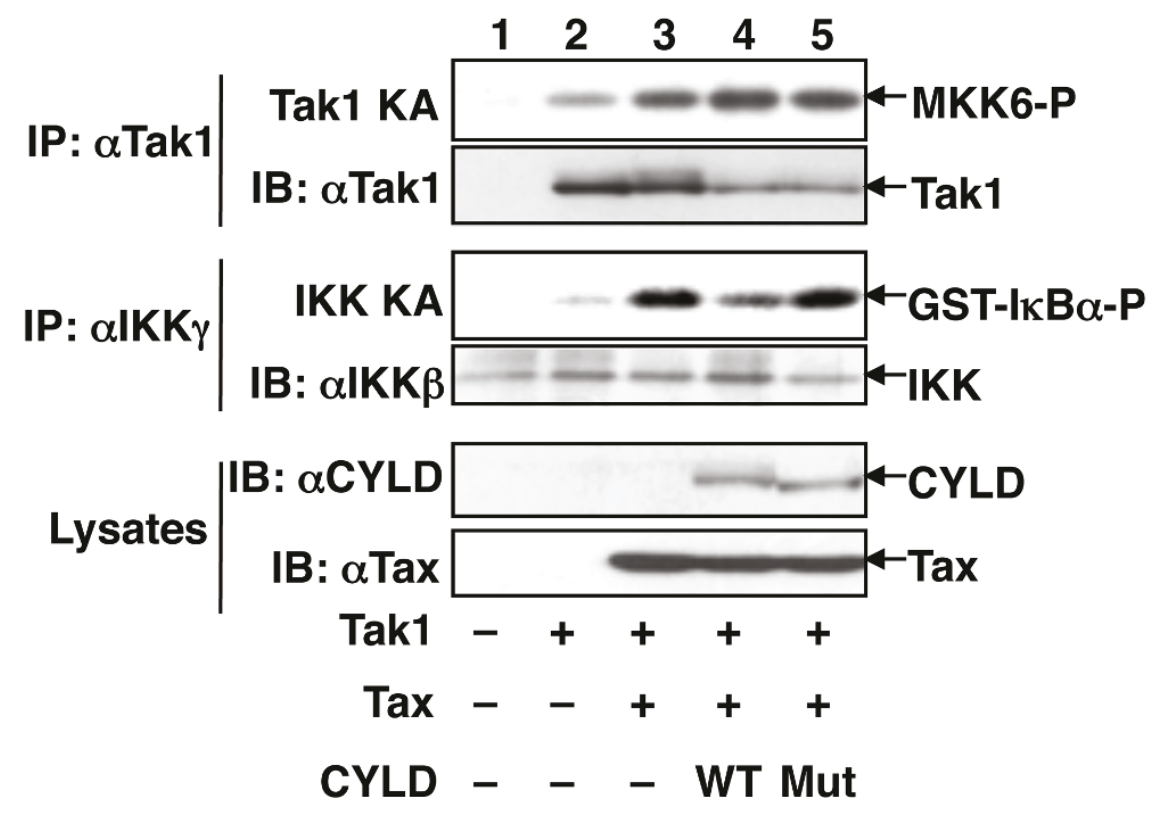

Figure 3 CYLD inhibits Tax-stimulated activation of IKK but not that of Tak1. HEK293 cells were transfected with the indicated expression vectors. Cell lysates were subjected to in vitro kinase assays to detect the activation of endogenous Tak1 (panel 1) and IKK (panel 3). Following autoradiography, the kinase assay membranes were subjected to IB to detect the Tak1 (panel 2) and IKK $\beta$ (panel 4) proteins. The expression level of CYLD and Tax was monitored by IB (panels 5 and 6). 


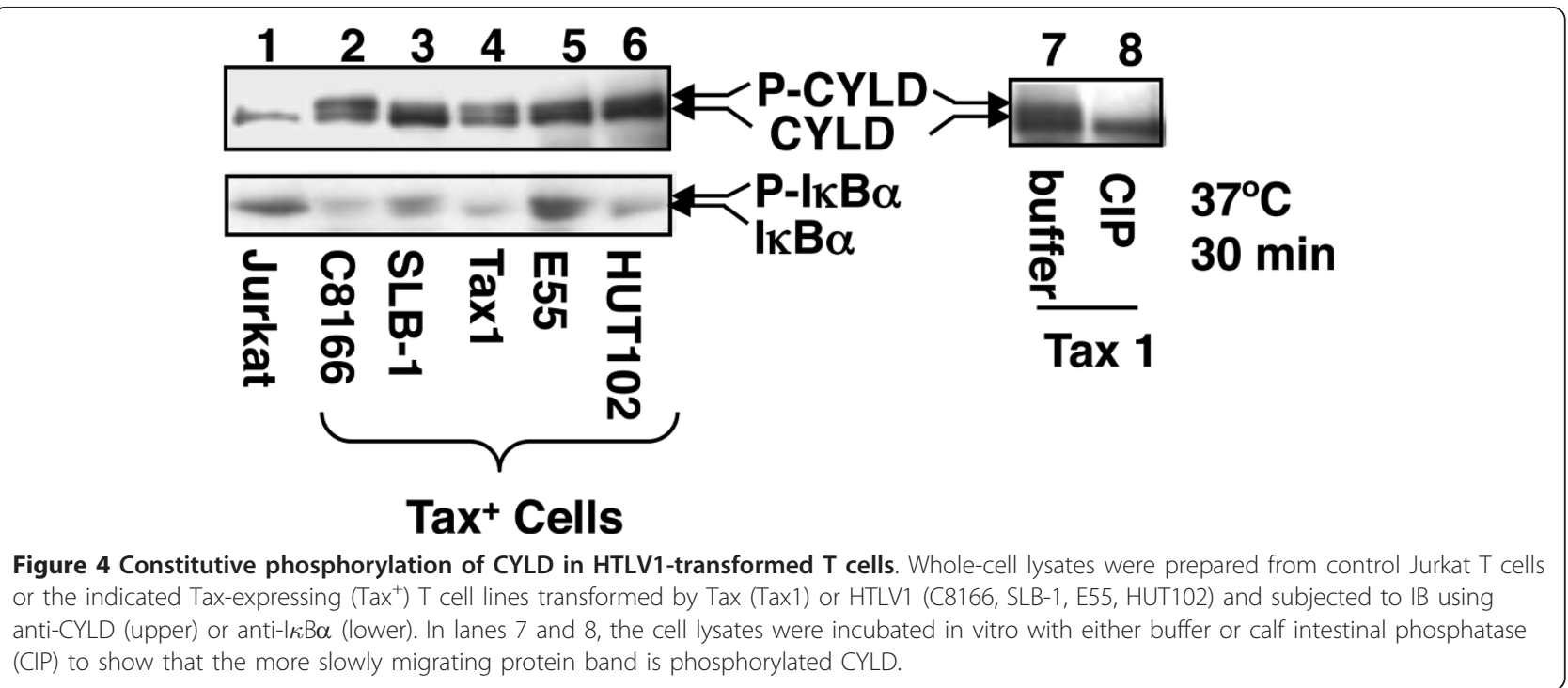

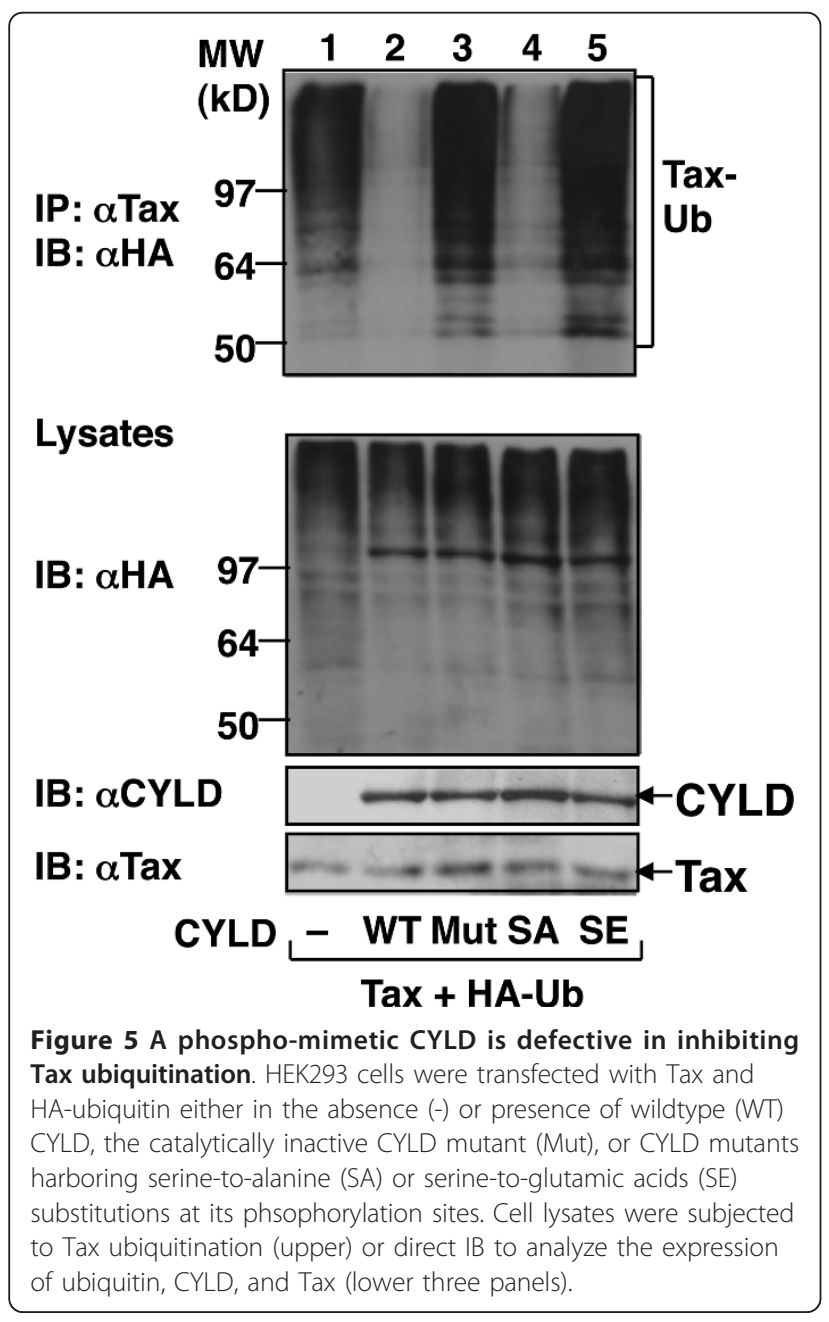

which may contribute to the aberrant activation of IKK and NF- $\kappa$ B.

\section{Discussion}

The data presented in this paper demonstrate a role for CYLD in regulating the ubiquitination of Tax, oncoprotein of the leukemia virus HTLV1 [2]. CYLD is physically assembled into the Tax complex and involved in negative regulation of Tax ubiquitination and signaling function. Furthermore, Tax and CYLD appear to mutually regulate, since CYLD is constitutively phosphorylated in HTLV1-transformed T cells.

Strong evidence suggests that Tax ubiquitination plays a critical role in its signaling function in the NF- $\kappa \mathrm{B}$ pathway [35]. Indeed, we have shown that CYLDmediated Tax deubiquitination is associated with attenuation of IKK activation. Similarly, the ubiquitination and function of Tax are also regulated by another deubiquitinase, USP20 [36]. These findings suggest that ubiquitinated Tax may be targeted by different deubiquitinases, although precisely how the different deubiquitinases regulate Tax during HTLV1 infection remains to be further studied.

Our current study demonstrates that the CYLDmediated Tax deubiquitination did not affect Tax's ability to activate Tak1. This finding suggests that Tak1 activation is insufficient for Tax-mediated IKK activation, implicating the involvement of different mechanisms in Tax activation of Tak1 and IKK. In further support of this idea, our previous work demonstrates that a Tax mutant, TaxM22, is capable of Tak1 activation despite its defect in IKK activation [34]. One possibility is that Tax not only activates Tak1 but also 
recruits Tak1 to the IKK complex to mediate IKK activation. Future studies will examine whether Tax ubiquitination is required for recruiting Tak1 to IKK.

The catalytic activity of CYLD is negatively regulated by its phosphorylation [22]. Cellular stimuli induce transient phosphorylation of CYLD, which may contribute to the optimal activation of IKK. A remarkable finding in the present study is that CYLD is constitutively phosphorylated in a large panel of HTLV1-transformed T cells. Since a phospho-mimetic CYLD mutant is defective in inhibiting Tax ubiquitination, the CYLD phosphorylation may contribute to the chronic activation of IKK and NF- $\kappa$ B in HTLV1-transformed T cells. We have previously shown that induction of CYLD phosphorylation by TNF- $\alpha$ or mitogens is mediated by the IKK complex [22]. However, we found that in HEK293 cells, Tax was insufficient for the induction of CYLD phosphorylation (data not shown). This result could be due to the low expression level of IKK components, particularly IKK $\gamma$ [37], although the possibility for involvement of additional signaling factors cannot be excluded. Regarding the latter possibility, a recent study suggests that CYLD phosphorylation can also be mediated by the IKK-related kinase IKK $\varepsilon$ and contributes to $\mathrm{IKK} \varepsilon$-induced tumorigenesis [38]. Whether IKKع is involved in the CYLD phosphorylation in HTLV1-trnasformed $\mathrm{T}$ cells is yet to be investigated. It also remains to be examined whether CYLD phosphorylation also contributes to HTLV1-induced T-cell transformation. Nevertheless, our data suggest that CYLD phosphorylation is a mechanism that mediates constitutive Tax ubiquitination and signaling function in HTLV1-transformed T cells.

\section{Conclusions}

The results of this study demonstrate a role for the DUB CYLD in the negative regulation of HTLV1 Tax protein. CYLD inhibits Tax-stimulated IKK activation via deubiquitinating Tax, although the CYLD-mediated Tax deubiquitination does not affect Tax activation of Tak1. Our data further suggest that HTLV1 has developed a mechanism to override the negative-regulatory role of CYLD, and this mechanism involves the induction of CYLD phosphorylation.

\section{Methods}

\section{Cell lines and transfection}

Human embryonic kidney cell line 293, human leukemia T-cell line Jurkat, and HTLV1-transformed human Tcell lines were described previously [34]. The Tax1 cell line is an IL-2-dependent human T-cell line immortalized by HTLV1 Tax in the context of a herpes saimiri vector [39]. For transient transfection, 293 cells were seeded in 6-well plates and transfected using Lipofectamine-2000 (Invitrogen).
Plasmid constructs, antibodies, and other reagents pCMV4-Tax was kindly provided by Dr. Warner Greene [40]. pcDNA-based expression vectors encoding HAtagged IKK $\gamma$, Ubiquitin, CYLD, a catalytically inactive CYLD mutant (CYLDmut) were as described [14,22,41]. CYLD SA is a phosphorylation-deficient mutant harboring alanine substitutions of seven serine residues, and CYLD SE is a phospho-mimetic CYLD mutant harboring serine to glutamic acid substitutions [22]. Ubiquitin mutants K48 and K63 (provided by Dr. Zhijian Chen) carry lysine-toarginine substitutions in all lysine residues, except K48 and K63, respectively. Ubiquitin mutants K48R and K63R harbor mutations in lysine 48 and 63, respectively. pCMVHA-Tak1 expression vector and anti-Tak1 antibody were kindly provided by Drs. Kunihiro Matsumoto and Jun Ninomiya-Tsuji [42]. All other reagents were described previously [34].

Immunoblotting (IB), immunoprecipitation (IP), in vitro kinase assay, and ubiquitination assay

Cell lysates were prepared by lysing the cells in RIPA buffer for IB and IP [43]. For kinase assays, cells were lysed in a kinase lysis buffer supplemented with phosphatase inhibitors and immediately subject to in vitro kinase assays as described previously [9]. For ubiquitination assays, cells were transfected with HA-tagged ubiquitin or its mutants along with other expression vectors. The cells were lysed in RIPA buffer supplemented with $4 \mathrm{mM} \mathrm{N}$-ethylmaleimide (NEM) and immediately bioled for $5 \mathrm{~min}$ in the presence of $1 \%$ SDS and then diluted 10 times with RIPA buffer. Ubiquitinated Tax was isolated by IP using antiTax and detected by IB with anti-HA.

\section{Immunofluorescence assay}

HEK293 cells were transiently transfected with HACYLD and Tax and subjected to indirect immunofluorescence assays as described [18].

\section{List of abbreviations}

HTLV1: human T-cell leukemia virus type 1; IKK: IKB kinase; DUB: deubiquitinase; IB: immunoblotting; IP: immunoprecipitation; NEM: Nethylmaleimide

\section{Acknowledgements}

We thank W.C. Greene for Tax expression vectors, Drs. Jun Niomiya-Tsuji and Kunihiro Matumoto for Tak1 antibody and expression vector, and AIDS Research and Reference Program of NIAID for anti-Tax hybridoma. This study was supported by National Institutes of Health grant Al064639.

\footnotetext{
Author details

'Laboratory of Gene Regulation and Signal Transduction, Department of Pharmacology, School of Medicine, University of California at San Diego, La Jolla, CA 92093, USA. ²Department of Melanoma Medical Oncology, The University of Texas MD Anderson Cancer Center, 7455 Fannin Street, Box 902, Houston TX 77030, USA. ${ }^{3}$ Department of Immunology, The University of Texas MD Anderson Cancer Center, 7455 Fannin Street, Box 902, Houston TX 77030, USA.
} 


\section{Authors' contributions}

XW and MZ designed and carried out the experiments and analyzed the data. SCS wrote the manuscript. All authors read and approved the final manuscript.

\section{Competing interests}

The authors declare that they have no competing interests.

Received: 23 June 2011 Accepted: 8 August 2011

Published: 8 August 2011

\section{References}

1. Yoshida M: Multiple viral strategies of HTLV-1 for dysregulation of cell growth control. Annu Rev Immunol 2001, 19:475-496.

2. Matsuoka M: Human T-cell leukemia virus type I (HTLV-I) infection and the onset of adult T-cell leukemia (ATL). Retrovirology 2005, 2:27.

3. Shuh M, Beilke M: The human T-cell leukemia virus type 1 (HTLV-1): new insights into the clinical aspects and molecular pathogenesis of adult T-cell leukemia/lymphoma (ATLL) and tropical spastic paraparesis/HTLV-associated myelopathy (TSP/HAM). Microsc Res Tech 2005, 68:176-196.

4. Matsuoka M, Jeang KT: Human T-cell leukaemia virus type 1 (HTLV-1) infectivity and cellular transformation. Nat Rev Cancer 2007, 7:270-280

5. Sun SC, Yamaoka S: Activation of NF-KB by HTLV-I and implications for cell transformation. Oncogene 2005, 24:5952-5964

6. Karin M, Ben-Neriah Y: Phosphorylation meets ubiquitination: the control of NF-[kappa]B activity. Annu Rev Immunol 2000, 18:621-663.

7. Hayden MS, Ghosh S: Shared principles in NF-kappaB signaling. Cell 2008, 132:344-362.

8. Chu Z-L, DiDonato JA, Hawiger J, Ballard DW: The Tax oncoprotein of human T-cell leukemia virus type 1 associates with and persistently activates IKB kinases containing IKKa and IKKß.J Biol Chem 1998, 273:15891-15894.

9. Uhlik M, Good L, Xiao G, Harhaj EW, Zandi E, Karin M, Sun S-C: NF-kappaBinducing kinase and IkappaB kinase participate in human T-cell leukemia virus I Tax-mediated NF-kappaB activation. J Biol Chem 1998, 273:21132-21136.

10. Yin M-J, Christerson LB, Yamamoto Y, Kwak Y-T, Xu S, Mercurio F, Barbose M, Cobb MH, Gaynor RB: HTLV-I Tax protein binds to MEKK1 to stimulate IkB kinase activity and NF-KB activation. Cell 1998, 93:875-884

11. Fu J, Qu Z, Yan P, Ishikawa C, Ageilan Rl, Rabson AB, Xiao G: The tumor suppressor gene WWOX links the canonical and noncanonical NF-KB pathways in HTLV-I Tax-mediated tumorigenesis. Blood 2011, 117:1652-1661.

12. Qu Z, Xiao G: Human T-Cell Lymphotropic Virus: A Model of NF-KBAssociated Tumorigenesis. Viruses 2011, 3:714-749.

13. Chu Z-L, Shin Y-A, Yang J-M, DiDonato JA, Ballard DW: IKKY mediates the interaction of cellular IKB kinases with the Tax transforming protein of human T cell leukemia virus type 1. J Biol Chem 1999, 274:15297-15300.

14. Harhaj EW, Sun S-C: IKKY serves as a docking subunit of the IKB kinase and mediates interaction of IKK with the human T-cell leukemia virus Tax protein. J Biol Chem 1999, 274:22911-22914.

15. Jin D-Y, Giordano V, Kibler KV, Nakano H, Jeang K-T: Role of adaptor function in oncoprotein-mediated activation of NF-KB: HTLV-I Tax interacts directly with IKB kinase g. J Biol Chem 1999, 274:17402-17405

16. Lamsoul I, Lodewick J, Lebrun S, Brasseur R, Burny A, Gaynor RB, Bex F: Exclusive ubiquitination and sumoylation on overlapping lysine residues mediate NF-kappaB activation by the human T-cell leukemia virus tax oncoprotein. Mol Cell Biol 2005, 25:10391-10406.

17. Nasr R, Chiari E, El-Sabban M, Mahieux R, Kfoury Y, Abdulhay M, Yazbeck V, Hermine $\mathrm{O}, \mathrm{dT} \mathrm{H}$, Pique $\mathrm{C}$, Bazarbachi A: Tax ubiquitylation and sumoylation control critical cytoplasmic and nuclear steps of NF-kappaB activation. Blood 2006, 107:4021-4029.

18. Harhaj NS, Sun SC, Harhaj EW: Activation of NF-kappa B by the human T cell leukemia virus type I (HTLV-I) tax oncoprotein is associated with ubiquitin-dependent relocalization of IKK. J Biol Chem 2007 , 282:4185-4192.

19. Liu S, Chen ZJ: Expanding role of ubiquitination in NF-KB signaling. Cell Res 2011, 21:6-21.
20. Sun SC: Deubiquitylation and regulation of the immune response. Nat Rev Immunol 2008, 8:501-511.

21. Komander D, Lord CJ, Scheel H, Swift S, Hofmann K, Ashworth A, Barford D: The structure of the CYLD USP domain explains its specificity for Lys63linked polyubiquitin and reveals a B Box module. Mol Cell 2008, 29:451-464.

22. Reiley W, Zhang M, Wu X, Graner E, Sun S-C: Regulation of the deubiquitinating enzyme CYLD by IkappaB kinase gamma-dependent phosphorylation. Mol Cell Biol 2005, 25:3886-3895.

23. Shembade N, Harhaj NS, Yamamoto M, Akira S, Harhaj EW: The human Tcell leukemia virus type 1 Tax oncoprotein requires the ubiquitinconjugating enzyme Ubc13 for NF-kappaB activation. J Virol 2007, 81:13735-13742.

24. Kovalenko A, Chable-Bessia C, Cantarella G, Israel A, Wallach D, Courtois G: The tumour suppressor CYLD negatively regulates NF-kappaB signalling by deubiquitination. Nature 2003, 424:801-805.

25. Trompouki E, Hatzivassiliou E, Tsichritzis T, Farmer H, Ashworth A, Mosialos G: CYLD is a deubiquitinating enzyme that negatively regulates NF-kappaB activation by TNFR family members. Nature 2003, 424:793-796.

26. Brummelkamp TR, Nijman SM, Dirac AM, Bernards R: Loss of the cylindromatosis tumour suppressor inhibits apoptosis by activating NFkappaB. Nature 2003, 424:797-801.

27. Ninomiya-Tsuji J, Kishimoto K, Hiyama A, Inoue J, Cao Z, Matsumoto K: The kinase TAK1 can activate the NIK-I kappaB as well as the MAP kinase cascade in the IL-1 signalling pathway. Nature 1999, 398:252-256.

28. Takaesu G, Surabhi RM, Park KJ, Ninomiya-Tsuji J, Matsumoto K, Gaynor RB: TAK1 is critical for lkappaB kinase-mediated activation of the NF-kappaB pathway. J Mol Biol 2003, 326:105-115.

29. Liu HH, Xie M, Schneider MD, Chen ZJ: Essential role of TAK1 in thymocyte development and activation. Proc Natl Acad Sci USA 2006, 103:11677-11682.

30. Sato S, Sanjo H, Takeda K, Ninomiya-Tsuji J, Yamamoto M, Kawai T, Matsumoto K, Takeuchi O, Akira S: Essential function for the kinase TAK1 in innate and adaptive immune responses. Nat Immunol 2005, 6:1987-1095.

31. Shim JH, Xiao C, Paschal AE, Bailey ST, Rao P, Hayden MS, Lee KY, Bussey C, Steckel M, Tanaka N, et al: TAK1, but not TAB1 or TAB2, plays an essential role in multiple signaling pathways in vivo. Genes Dev 2005, 19:2668-2681.

32. Wan YY, Chi H, Xie M, Schneider MD, Flavell RA: The kinase TAK1 integrates antigen and cytokine receptor signaling for T cell development, survival and function. Nat Immunol 2006, 7:851-858,

33. Shambharkar PB, Blonska M, Pappu BP, Li H, You Y, Sakurai H, Darnay BG, Hara H, Penninger J, Lin X: Phosphorylation and ubiquitination of the IkappaB kinase complex by two distinct signaling pathways. EMBO J 2007, 26:1794-1805.

34. Wu X, Sun SC: Retroviral oncoprotein Tax deregulates NF-KB by activating Tak1 and mediating Tak1-IKK physical association. EMBO Rep 2007, 8:510-515.

35. Sun SC, Cesarman E: NF-kB as a Target for Oncogenic Viruses. Curr Top Microbiol Immunol 2011, 349:197-244.

36. Yasunaga J, Lin FC, Lu X, Jeang K-T: Ubiquitin-specific peptidase 20 targets TRAF6 and human T cell leukemia virus type I Tax to negatively regulate NF-KB signaling. J Virol 2011, 85:6212-6219.

37. Xiao G, Harhaj EW, Sun S-C: Domain-specific interaction with IKKY is an essential step in Tax-mediated activation of IKK. J Biol Chem 2000 275:34060-34067.

38. Hutti JE, Shen RR, Abbott DW, Zhou AY, Sprott KM, Asara JM, Hahn WC, Cantley LC: Phosphorylation of the tumor suppressor CYLD by the breast cancer oncogene IKKepsilon promotes cell transformation. Mol Cell 2009, 34:461-472.

39. Grassmann R, Berchtold S, Radant I, Alt M, Fleckenstein B, Sodroski JG, Haseltine WA, Ramstedt U: Role of human T-cell leukemia virus type I X region proteins in immortalization of primary human lymphocytes in culture. J Virol 1992, 66:4570-4575.

40. Smith MR, Greene WC: Identification of HTLV-1 tax transactivator mutants exhibiting novel transcriptional phenotypes. Genes Dev 1990, 4:1875-1885.

41. Xiao G, Harhaj EW, Sun SC: NF-kappaB-inducing kinase regulates the processing of NF-kappaB2 p100. Mol Cell 2001, 7:401-409. 
42. Shibuya H, Yamaguchi K, Shirakabe K, Tonegawa A, Gotoh Y, Ueno N, Irie K, Nishida E, Matsumoto K: TAB1: an activator of the TAK1 MAPKKK in TGFbeta signal transduction. Sciece 1996, 272:1179-1182.

43. Xiao G, Cvijic ME, Fong A, Harhaj EW, Uhlik MT, Waterfield M, Sun SC: Retroviral oncoprotein Tax induces processing of NF-kappaB2/p100 in T cells: evidence for the involvement of IKKalpha. EMBO J 2001, 20:6805-6815

doi:10.1186/2045-3701-1-27

Cite this article as: Wu et al:: Mutual regulation between deubiquitinase CYLD and retroviral oncoprotein Tax. Cell \& Bioscience 2011 1:27.

Submit your next manuscript to BioMed Central and take full advantage of:

- Convenient online submission

- Thorough peer review

- No space constraints or color figure charges

- Immediate publication on acceptance

- Inclusion in PubMed, CAS, Scopus and Google Scholar

- Research which is freely available for redistribution

Submit your manuscript at www.biomedcentral.com/submit
C) Biomed Central 